\title{
The Drosophila Perlecan gene trol regulates multiple signaling pathways in different developmental contexts Jonathan R Lindner ${ }^{1}$, Paul R Hillman ${ }^{1}$, Andrea L Barrett ${ }^{1}$, Megan C Jackson ${ }^{2,3}$, Trinity L Perry ${ }^{1,4}$, Youngji Park ${ }^{1,5}$ and Sumana Datta*1,2
}

Address: ${ }^{1}$ Department of Biochemistry \& Biophysics, Texas A\&M University, College Station, Texas, 77843-2128, USA, ${ }^{2}$ Department of Biology, Texas A\&M University, College Station, Texas, 77843-2128, USA, ${ }^{3}$ Department of Small Animal Medicine and Surgery, College of Veterinary Medicine, Texas A\&M University, College Station, Texas, 77843-4474, USA, ${ }^{4}$ Department of Cell Biology and Molecular Genetics, University of Maryland, College Park, Maryland, 20742, USA and ${ }^{5}$ Division of Hematology and Oncology, 10900 Euclid Ave, WRB 2-104, Case Western Reserve University School of Medicine, Cleveland, Ohio, 44106, USA

Email: Jonathan R Lindner - jrlindner@tamu.edu; Paul R Hillman - prhillm@neo.tamu.edu; Andrea L Barrett - albarrett@tamu.edu; Megan C Jackson - carriermk@tamu.edu; Trinity L Perry - trinitylperry@hotmail.com; Youngji Park - yxp14@case.edu;

Sumana Datta* - sumad@tamu.edu

* Corresponding author

Published: 2 November 2007

BMC Developmental Biology 2007, 7:121 doi:10.1186/147|-213X-7-121
Received: 8 March 2007

Accepted: 2 November 2007

This article is available from: http://www.biomedcentral.com//47/-2/3X/7//2I

(c) 2007 Lindner et al; licensee BioMed Central Ltd.

This is an Open Access article distributed under the terms of the Creative Commons Attribution License (http://creativecommons.org/licenses/by/2.0), which permits unrestricted use, distribution, and reproduction in any medium, provided the original work is properly cited.

\begin{abstract}
Background: Heparan sulfate proteoglycans modulate signaling by a variety of growth factors. The mammalian proteoglycan Perlecan binds and regulates signaling by Sonic Hedgehog, Fibroblast Growth Factors (FGFs), Vascular Endothelial Growth Factor (VEGF) and Platelet Derived Growth Factor (PDGF), among others, in contexts ranging from angiogenesis and cardiovascular development to cancer progression. The Drosophila Perlecan homolog trol has been shown to regulate the activity of Hedgehog and Branchless (an FGF homolog) to control the onset of stem cell proliferation in the developing brain during first instar. Here we extend analysis of trol mutant phenotypes to show that trol is required for a variety of developmental events and modulates signaling by multiple growth factors in different situations.
\end{abstract}

Results: Different mutations in trol allow developmental progression to varying extents, suggesting that trol is involved in multiple cell-fate and patterning decisions. Analysis of the initiation of neuroblast proliferation at second instar demonstrated that trol regulates this event by modulating signaling by Hedgehog and Branchless, as it does during first instar. Trol protein is distributed over the surface of the larval brain, near the regulated neuroblasts that reside on the cortical surface. Mutations in trol also decrease the number of circulating plasmatocytes. This is likely to be due to decreased expression of pointed, the response gene for VEGF/PDGF signaling that is required for plasmatocyte proliferation. Trol is found on plasmatocytes, where it could regulate VEGF/PDGF signaling. Finally, we show that in second instar brains but not third instar brain lobes and eye discs, mutations in trol affect signaling by Decapentaplegic (a Transforming Growth Factor family member), Wingless (a Wnt growth factor) and Hedgehog.

Conclusion: These studies extend the known functions of the Drosophila Perlecan homolog trol in both developmental and signaling contexts. These studies also highlight the fact that Trol function is not dedicated to a single molecular mechanism, but is capable of regulating different growth factor pathways depending on the cell-type and event underway. 


\section{Background}

Heparan sulfate proteoglycans (HSPGs) are a family of cell-surface and extracellular proteins modified by the attachment of glycosaminoglycan chains. The general structure of the protein core determines the family the HSPG belongs to: Syndecans contain a transmembrane domain, Glypicans are tethered to the cell surface via a GPI linkage and Perlecans are secreted components of the extracellular matrix. Both the protein core and glycan chains play important roles in HSPG function through protein-protein and sugar-protein interactions. Genetic studies, first in Drosophila and later in mouse and zebrafish, demonstrated the importance of the heparan sulfate chains on all three types of HSPGs for signaling by multiple growth factors such as the Fibroblast Growth Factors (FGFs), Hedgehogs, Wnts and Transforming Growth Factors (TGF $\beta s$ ) (reviewed in [1]).

Perlecan is the largest member of the HSPG family with a core protein of approximately $450 \mathrm{kD}$ in size. Perlecan has been linked to signaling by the heparan-dependent growth factors FGF2, Vascular Endothelial Growth Factor (VEGF) and Sonic Hedgehog (SHH) in mammalian systems (reviewed in $[2,3]$ ). Studies of Perlecan knock-out mice have demonstrated roles for Perlecan in vascular development and chondrogenesis as well as maintenance of basement membrane integrity [4-7]. Additional mammalian studies have revealed Perlecan's functions in angiogenesis and carcinogenesis ([8-11], reviewed in [2,12]). Mutation of Perlecan in humans leads to the muscle tone symptoms of Schwartz-Jampel syndrome, possibly through altered excitability of the neuromuscular junction and the skeletal abnormalities of Silver-Handmaker syndrome, presumably through effects on chondrogenesis [13-15].

Studies of Perlecan in invertebrate model systems have led to additional insights into Perlecan function. The single Perlecan gene in C. elegans is encoded by the unc-52 locus [16]. Mutations in unc-52 result in embryonic or adult paralysis due to defects in body wall muscle cells $([16,17]$, reviewed in [18]). Mutations in unc-52 also enhance cell migration defects caused by decreased netrin, FGF, TGF $\beta$ or Wnt signaling. In Drosophila, Perlecan is encoded by the trol gene on the X chromosome $[19,20]$, which was initially implicated in the control of stem cell division in the developing larval brain $[21,22]$. In the larval brain, trol promotes the cell cycle progression of mitotically arrested neuroblasts $[23,24]$ through modulation of FGF and Hedgehog signaling [19]. These Drosophila studies were the first to link Perlecan to Hedgehog signaling. More recently, studies of oogenesis in Drosophila have uncovered a role for Perlecan in the maintenance of epithelial cell polarity through interactions with the extracellular matrix receptor Dystroglycan [25].
The many signaling pathways associated with HSPGs in general and Perlecan in particular led us to ask what other biological processes may require Perlecan function. We used a series of trol mutants to investigate several phenotypes ranging from overall developmental progress to specific alterations of stem cell division and hemocyte production. Furthermore, analysis of signaling pathway response genes revealed that while mutations in Perlecan decrease signaling in multiple pathways, at least some of these effects are tissue specific.

\section{Results and discussion \\ Development and lethal phase}

We had previously shown that the viable trolb22 and the lethal $\mathrm{trol}^{8}$, trol ${ }^{4}$, and trolsd alleles form an allelic series of increasing severity based on their onset of neuroblast proliferation phenotype in first instar larval brain lobes [24]. Identification [19] and phenotypic analysis of a fifth trol allele, $\mathrm{trol}^{7}$, revealed that $\mathrm{trol}^{7}$ is the strongest allele with respect to the first instar proliferation phenotype (Fig. 1A). Unexpectedly, trol ${ }^{7}$ mutant larvae appeared healthier overall than other trol mutant larvae, suggesting that the order of allelic severity determined by analysis of first instar brain lobes would be different from one based on developmental progression. To test this hypothesis, we examined the lethal stage and developmental progression of larvae mutant for $\mathrm{trol}^{\mathrm{b} 22}$, $\mathrm{trol}^{8}, \mathrm{trol}^{4}, \mathrm{trols}^{\mathrm{sd}}$ and $\mathrm{trol}^{7}$. In all the experiments, crosses were designed to use sibling controls in order to minimize the effects of genetic background, which can be significant in fly stocks kept in reproductive isolation from each other for years in our laboratory. For the lethal trol alleles, $y$ trol ${ }^{x}$ Binsn stocks were used as the source of mutant and control larvae. At this stage of first instar, mutations in $y$ produce one of the few reliable phenotypic markers. Thus trol mutant animals were identified as $y$ mutant larvae that are $y$ trol $^{x}$ hemizygous males and sibling controls were a mixed population of $y^{+}$animals: $y$ trol $/$ Binsn heterozygotes, Binsn homozygous females and Binsn hemizygous males. Note that while Binsn homozygous females and hemizygous males can become viable adults, not all Binsn/Binsn or Binsn/Y larvae reach adulthood. Thus our comparison provides a measure of developmental progression and lethal phase that will err on the side of minimizing the trol mutant phenotype. For analysis of the viable trol ${ }^{b 22}$ allele, additional crosses were required to produce wild-type sibling controls from the homozygous $y$ trolb22 stock. $y$ trolb22 animals were crossed to the wild-type strain Canton Special (CS) to produce trolb22/CS heterozygous females. These females were mated to CS males to generate hemizygous $y$ trolb22 male larvae and $y^{+}$sibling control larvae (a mixture of heterozygous $y$ trolb22/CS female, homozygous CS female and hemizygous CS male) for the developmental studies. One hundred mutant and sibling control animals for each allele were collected at early first 
instar and monitored at 24 hour intervals for developmental progression and viability. Of these, only 1 mutant trol $^{4}$ and no trolsd animals pupariated. However, when the same numbers of $\mathrm{trol}^{\mathrm{b} 22} \mathrm{trol}^{8}$ and $\mathrm{trol}^{7}$ mutant larvae were analyzed and compared to sibling controls, 102\%, 38\% and $23 \%$ of the animals were able to pupariate, respectively (Fig. 1B). The pupariation assay resulted in shifts of perceived functional severity for both $\mathrm{trol}^{4}$ and $\mathrm{trol}^{7}$, with $\mathrm{trol}^{4}$ appearing stronger and $\mathrm{trol}^{7}$ appearing weaker.

Why would animals mutant for trol $^{7}$ (that has a strong effect on neuroblast proliferation) be able to progress further in development than animals mutant for $\mathrm{trol}^{4}$ which causes a weaker neuroblast proliferation phenotype? One possibility is that trol modulates the activity of different signaling pathways in different tissues. For example, a mutation that affects the ability of Trol to function in the Hh pathway would have a severe effect on developmental decisions that require $\mathrm{Hh}$ activity and very little effect on decisions that do not require Hh signaling. To address this possibility we investigated the impact of trol mutations on two distinct developmental events and several signaling pathways.

\section{Effects of trol mutations on $T N b$ proliferation}

trol was initially identified as a mutation on the X chromosome that affected the proliferation pattern of neuroblasts in the brain lobes and ventral ganglion $[21,22]$. Since neuroblasts in the thoracic region of the ventral ganglion begin proliferation in early second instar $[21,26,27]$, we evaluated the ability of thoracic neuroblasts (TNbs) to enter $\mathrm{S}$ phase in trol mutant animals. We adapted the idea of phenotypic classes to produce a scale for the extent of $\mathrm{TNb}$ proliferation at four hours post molt (Fig. 1C-G). Five TNb classes were defined as follows: Class 1, no neuroblasts labeled; Class 2, a small number of labeled neuroblasts with no distinct segmental pattern; Class 3, labeled neuroblasts in a segmentally repeated lines with very few labeled neuroblasts in between the lines or in the medial region of the ventral ganglion; Class 4, labeled neuroblasts in a segmentally repeated line with some labeled neuroblasts in between the lines or in the medial region of the ventral ganglion; and Class 5, labeled neuroblasts in heavily populated segmental pattern with many labeled neuroblasts in the medial portion of the ventral ganglion. When both sides of a ventral ganglion did not conform to a single class, the sample was scored as the higher class. This will have a conservative effect of scoring a partial loss-of-proliferation TNb phenotype as more wild-type. Thus we can have greater confidence in the significance of $\mathrm{TNb}$ proliferation phenotypes observed compared to controls.

We first examined the onset of TNb proliferation in wildtype sibling controls to determine the time point at which to assay the trol mutants (data not shown). In our hands, high levels of 5-Bromodeoxyuridine (BrdU)-labeled TNbs were first observed in control samples between 2-5 hours post molt depending on genetic background. This timing is slightly earlier than the previous observation that $\mathrm{TNb}$ mitosis begins between 28-34 hours post hatching, or 410 hours post molt (pm) to second instar [26]. To evaluate the $\mathrm{TNb}$ proliferation phenotype produced by the different trol alleles, at least twenty samples for each mutant and sibling control (generated as described above) were allowed to incorporate BrdU from 4-5 hours pm and scored for TNb class. The average score and standard error of the mean were calculated for each group of sibling controls. The control value for each study was set to a value of 4 to control for genetic background effects between experiments. Setting controls to a value of 4 on our 5 point scale was chosen to allow evaluation of over-proliferation $(>4)$ as well as under-proliferation $(<4)$ mutant phenotypes. To obtain the TNb phenotype score for each mutant allele we normalized the score for each sample to the respective sibling control and calculated the average and standard error of the mean (Table 1). Surprisingly, trolb22 mutants had a significantly higher than normal level of $\mathrm{TNb}$ proliferation ( $\mathrm{TNb}$ score $>4$ ) while the remaining trol mutants showed decreased $\mathrm{TNb}$ cell division compared to controls. The differences between mutant and control BrdU incorporation were statistically significant $(\mathrm{p}<$ 0.05) for each mutant allele. Comparison between mutants showed a phenotypic trend from trol ${ }^{b 22}$ having hyperactive $\mathrm{TNb}$ proliferation to trols as the mutant with the fewest labeled TNbs. In this assay $\mathrm{trol}^{7}$ mutants appear to have a weaker phenotype than trolsd.

\section{trol affects $\mathrm{Bnl}$ and Hh signaling in the ventral ganglia}

To determine if trol affects TNb proliferation through modulation of $\mathrm{Bnl}$ and $\mathrm{Hh}$ signaling, we used genetic interaction studies with the weak trol allele trolb22. As we have shown, trol ${ }^{b 22}$ animals have over proliferation of TNbs compared to sibling controls (Table 1). For the genetic interaction assay, $y$ trol $^{b 22}$ females were crossed to $b n l^{06916} / \mathrm{TM} 3 y^{+}$males to generate $y$ larvae that were $y$ trolb22 ; bnl06916/+ and $\gamma^{+}$sibling controls that were a combination of $y$ trol $^{b 22} ;+/ T M 3 y^{+}$males, $y$ trol $^{b 22} /+;+/ T M 3 y^{+}$ females and trolb22/+; bnl06916/+ females. None of the sibling controls had $\mathrm{TNb}$ proliferation scores outside of the normal (CS) range at this timepoint. $y$ trol $^{622}$ males carrying a single copy of the bn ${ }^{06916}$ allele had fewer BrdU labeled TNbs at 2-3 hours post molt to second instar (pm) compared to siblings that were hemizygous or heterozygous for trolb22 alone or heterozygous for both trolb22 and $b n l^{06916}$ (Fig. 2). The decreased TNb proliferation in samples heterozygous for bn $l^{06916}$ in a trol ${ }^{b 22}$ background compared to controls versus the increased proliferation in trol $^{b 22}$ animals wild-type for $b n l$ compared to controls sug- 


\section{A First Instar Nb proliferation phenotype}
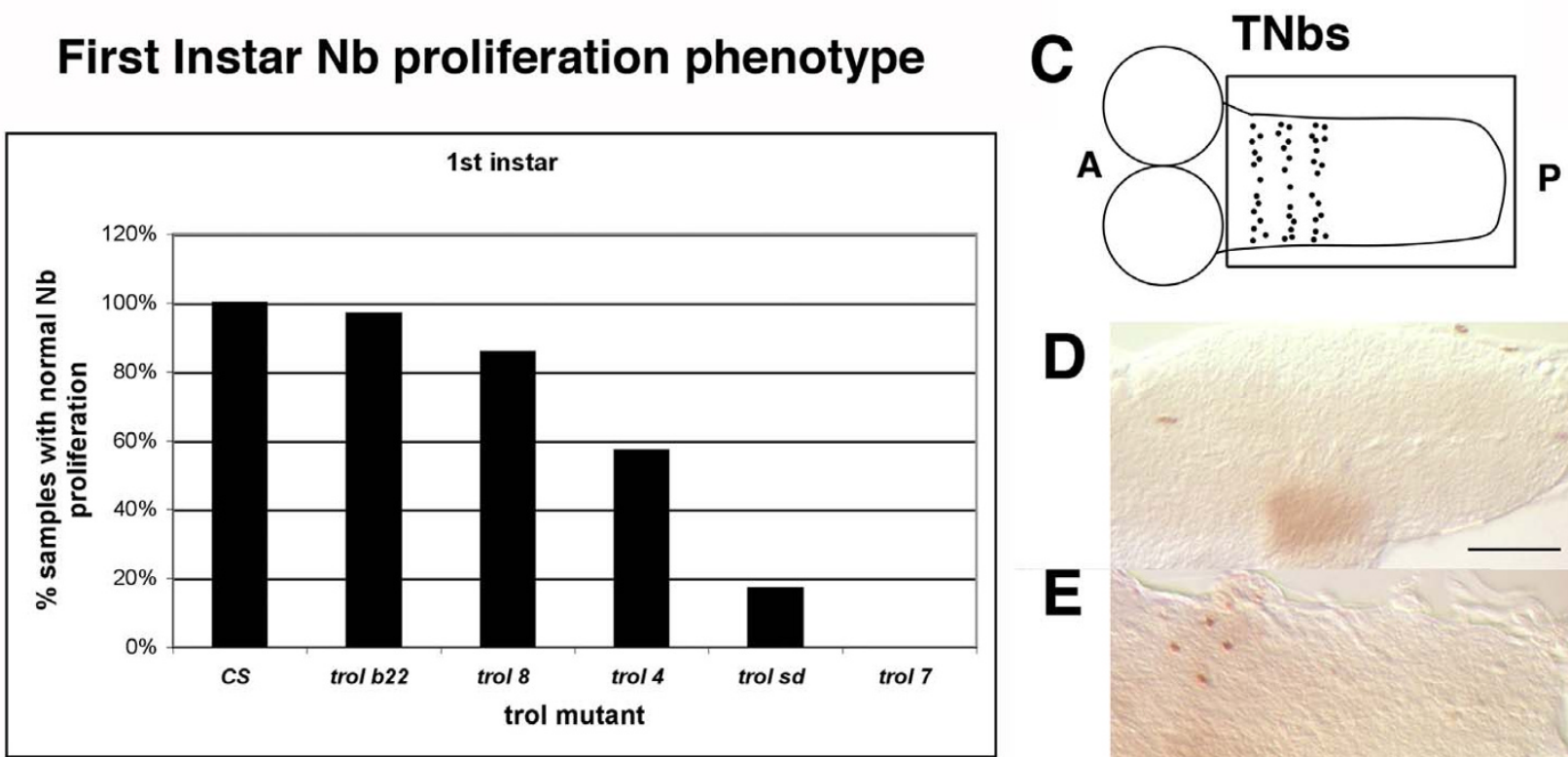

\section{B \% Progression to Third Instar or Pupae}

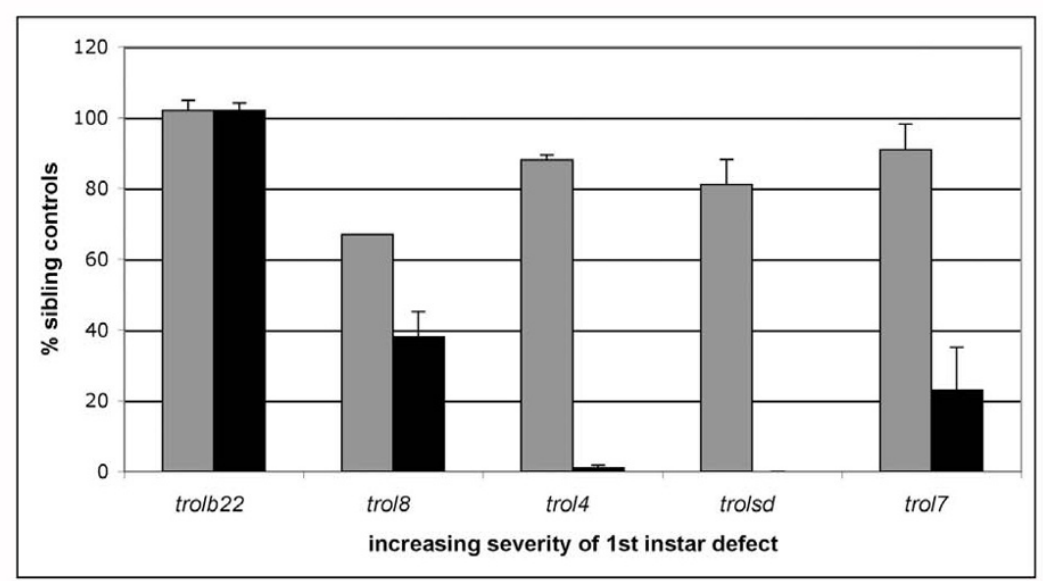

D

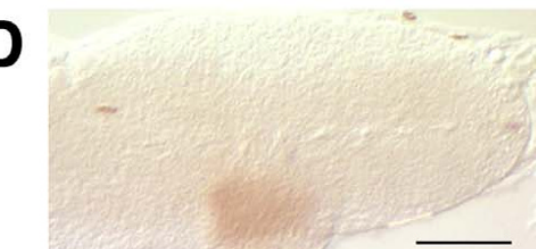

E

G

$\mathbf{F}$

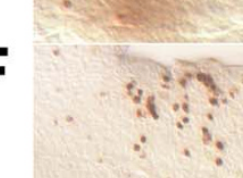

H

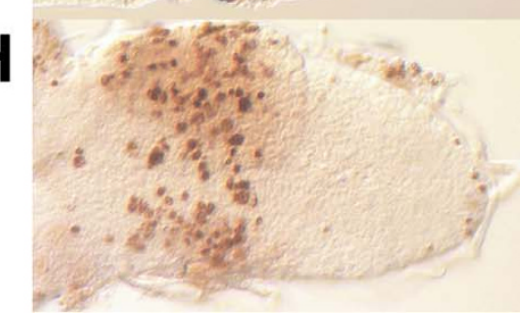

\section{Figure I}

Phenotypic series of trol alleles. A) First instar neuroblast proliferation phenotype presented as \% of samples with numbers of BrdU labeled neuroblasts falling within the control range, some data originally published in Park et al, 2003a. B) Lethal phase phenotype presented as the percentage of trol mutant animals capable of survival and development to third instar (grey bars) or to pupal formation (black bars) compared to sibling controls. Error bars indicate s.e.m. C) Cartoon of second instar larval brain with dividing TNBs in ventral ganglion. Boxed area indicates portion of brain shown in panels $D-G$ below. $A=$ anterior, $P=$ posterior. D-G) Examples of the five classes of BrdU incorporation into TNbs are shown. In all panels anterior is to the left, posterior is to the right. Scale bar in panel D indicates $25 \mathrm{um}$. D) None (class I). E) Few (class 2). F) Segmentally repeated lines with few extra neuroblasts (class 3). G) Segmentally repeated lines with several scattered neuroblasts (class 4). H) Heavily populated segmental pattern (class 5). 
Table I: TNb BrdU incorporation phenotype of trol mutants at 4-5 hours pm.

\begin{tabular}{ccc}
\hline trol allele & TNb score* & S.E.M. \\
\hline Control & 4.00 & 0.27 \\
trolb22 $^{\text {trol }}$ & 4.55 & 0.16 \\
trol $^{4}$ & 3.37 & 0.21 \\
trol & 3.00 & 0.27 \\
trolsd & 2.95 & 0.2 \\
& 2.45 & 0.21 \\
\hline
\end{tabular}

* All scores significantly different from controls at $\mathrm{p}<0.05$.

gests that the trol ${ }^{b 22}$ mutation affects signaling by Bnl in the ventral ganglion at second instar.

We also used genetic interactions to evaluate the possibility that trol might affect Hedgehog signaling in the ventral ganglion. For this study $y$ trol $^{b 22}$ females were crossed to $h h^{A C} / \mathrm{TM} y^{+}$males to generate $y$ larvae that were y trolb22; $h h^{A C} /+$ and $\gamma^{+}$sibling controls that were a combination of $y$ trol $^{b 22} ;+/ \mathrm{TM}^{2} y^{+}$males, $y$ trol $^{b 22} /+;+/ \mathrm{TM} 3 y^{+}$females and trol $^{b 22} /+; h h^{A C} /+$ females. trol ${ }^{b 22}$ animals carried a single copy of the $h h^{A C}$ allele, also had fewer dividing TNbs at 23 hours pm compared to sibling controls (Fig. 2). The decrease in the number of BrdU labeled TNbs in trolb22 hemizygotes upon heterozygosity for $h h^{A C}$ suggest that mutations in trol also weaken the signaling action of $\mathrm{Hh}$ in the ventral ganglion. To further test our hypotheses, we examined the signaling activity of $\mathrm{Bnl}$ and $\mathrm{Hh}$ in trol mutants directly by quantitative RealTime PCR (qRTPCR) in the central nervous system (CNS). To avoid interfering signals from the lobes of the second instar brain that might overwhelm differences in signal in the ventral ganglion, we isolated ventral ganglia from second instar trol mutant and sibling control brains at one hour post molt. First instar brains were dissected at 20 hours post hatching which correlates with the end of the BrdU labeling period used to assess neuroblast proliferation in first instar ([24] and this manuscript). RNA was isolated, cDNA synthesized and amplified and the level of expression of the Hh response gene ptc (Fig. 3) and the Bnl response gene pnt (Fig. 3) assayed. Our qRT-PCR data demonstrate that mutations in trol affect the strength of signaling by both $\mathrm{Hh}$ and Bnl in the larval ventral ganglion and in first instar larval brains (data not shown).

\section{Trol localization in the larval brain}

Previously we had isolated complexes containing either Trol and FGF2 or Trol and Hh by co-immunoprecipitation [19]. In combination with our genetic studies, these complexes suggested that the Trol protein regulates neuroblast division by binding growth factors that stimulate neuroblast proliferation in a manner similar to Perlecan-mediated promotion of ligand-receptor binding described in mammalian systems [28]. This model predicts that Trol protein should be localized near the regulated neuroblasts, i.e. the optic lobe and central brain neuroblasts of

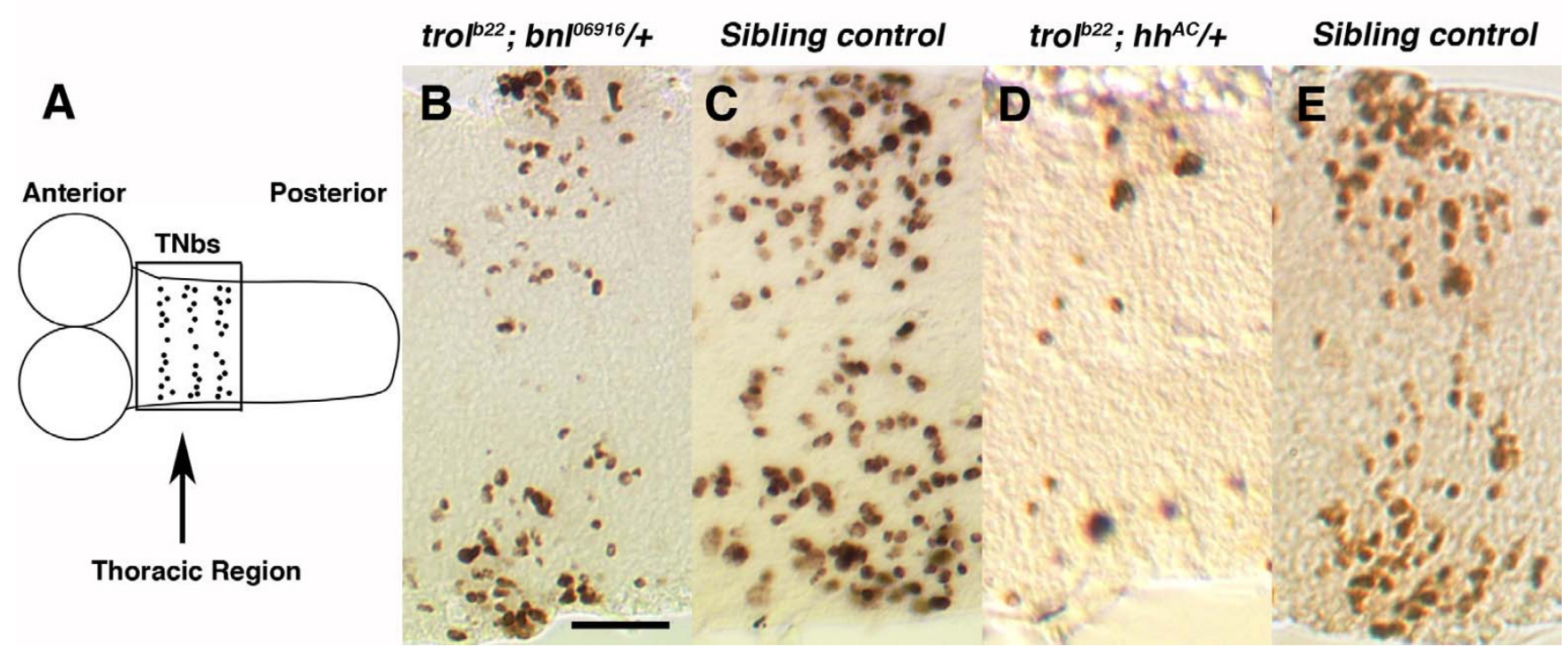

Figure 2

Trol modulates $\mathrm{Hh}$ and $\mathrm{Bnl}$ signaling in the ventral ganglion. A) Cartoon of second instar brain indicating location of TNbs. Boxed area outlines thoracic region shown in panels B-E. One hour BrdU incorporation in TNbs in B) a y trolb22; bn/06916/

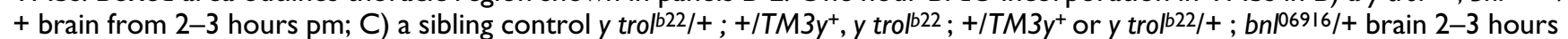

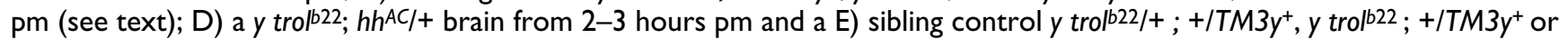
$y$ trolb22/+ ; hh $A C /+$ brain from 2-3 hours pm (see text). Scale bar in panel A indicates 10 um. 


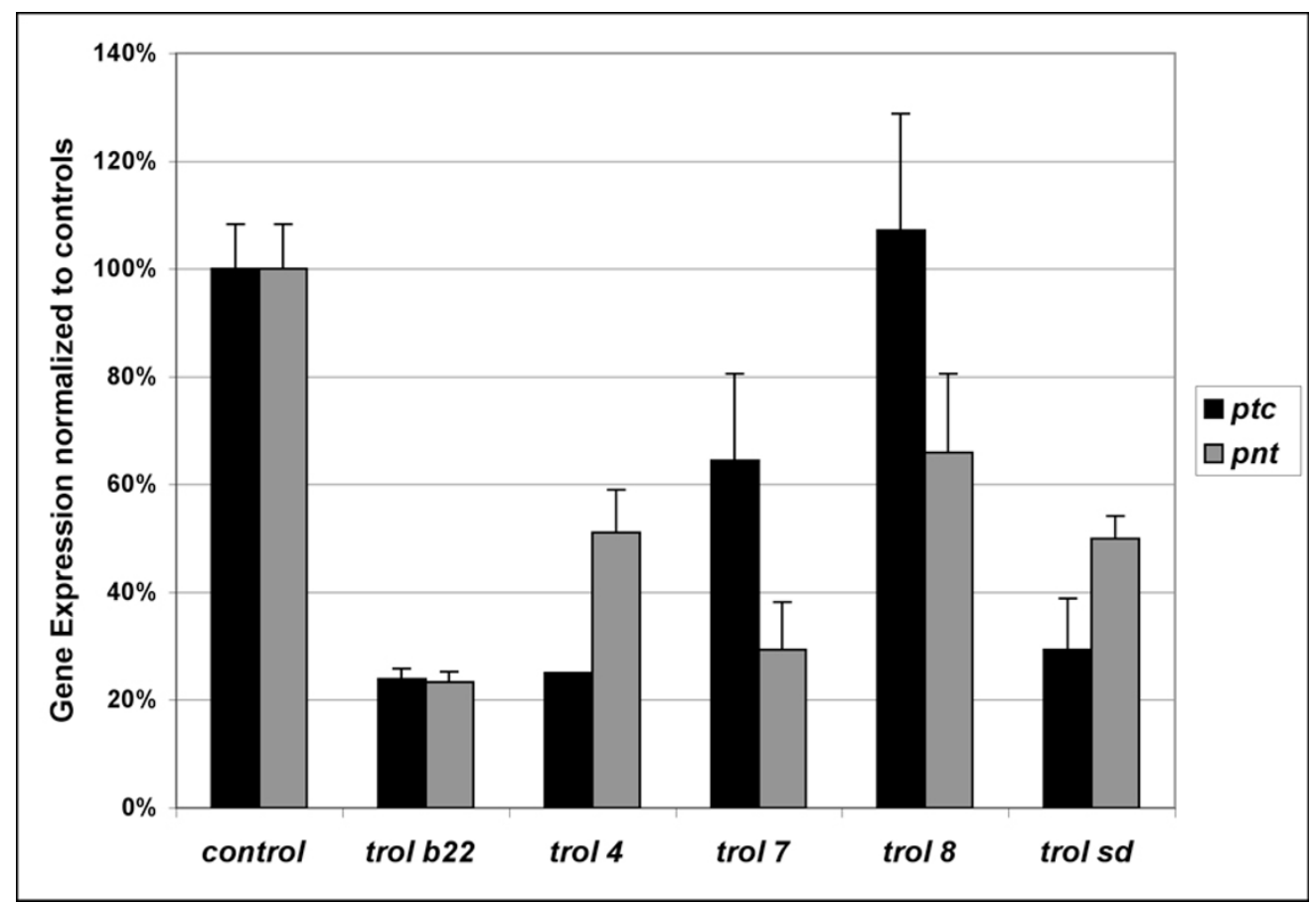

Figure 3

$\mathrm{Hh}$ and Bnl signaling activity in the ventral ganglion of trol mutant animals. Quantitative RT-PCR analysis of the expression of the $\mathrm{Hh}$ response gene ptc (black bars) and the $\mathrm{Bnl}$ response gene pnt (grey bars) in the ventral ganglia of trol mutant normalized to controls at one hour post molt to second instar. $\beta$-actin was used to as an internal control to normalize message levels. All analyses were done in triplicate and three different concentrations to ensure samples were within linear range of amplification. Error bars indicate standard deviation.

the first instar brain $[19-24,29]$ and the thoracic region of the second instar brain ([22] and Fig. 4A). In contrast, in situ hybridization studies in the third instar larval brain by Voigt et al [20] had revealed that only a few isolated cells at a distance from the optic lobe proliferation centers express trol. This led the authors to suggest that Trol is unlikely to regulate neuroblast proliferation by promoting binding of FGF-type ligands to their receptors since this would require Trol protein localization near the responding cells. However, since Trol is a secreted protein with a long half-life, mRNA expression patterns may not accurately portray protein localization. In addition, Voigt et al conducted their in situ analysis at late third instar, 23 days after the activation of neuroblast division at late first or early second instar. Thus the expression pattern observed for trol message at late third instar may not reflect expression of trol at earlier larval stages. Furthermore, a study of trol mRNA localization by in situ hybridization in embryos showed either no obvious staining in the CNS [30] or expression in a small subset of glial cells in the CNS [20]. However, analysis of Trol protein localization with an anti-Trol antibody in embryos revealed localization to the basement membrane of the CNS [30]. This evidence further suggests that trol message patterns may not reflect Trol protein localization. To address the conflicting models, we took advantage of a Trol protein trap in which the GFP gene is inserted within the endogenous trol locus [31]. Analysis of GFP localization in larval brains demonstrates that Trol-GFP is found in a layer, presumably the basal lamina, encompassing the entire outer surface of the larval brain with little to no signal detectable at internal sites within the brain (Fig. 4B-E). Trol-GFP was also observed in the basal lamina surrounding nerves emanating from the larval brain. The distribution of Trol over the entire brain was further verified by immunohistochemistry using an anti-Trol antibody (Fig. 4F). This localization of the Trol protein is consistent with the model that Trol binds Bnl and $\mathrm{Hh}$ and facilitates their signaling to promote neuroblast proliferation, as the regulated neuroblasts are found at the surface of the cellular cortex in both the brain lobes and the ventral ganglion $[21,26,32]$. To determine if the localization of Trol-GFP to the basal lamina was unique to the larval brain, we examined Trol-GFP in the salivary glands. As in our larval brain studies, Trol-GFP is found on the surface of the gland, presumably as a component of the basal lamina (Fig. 4G). 


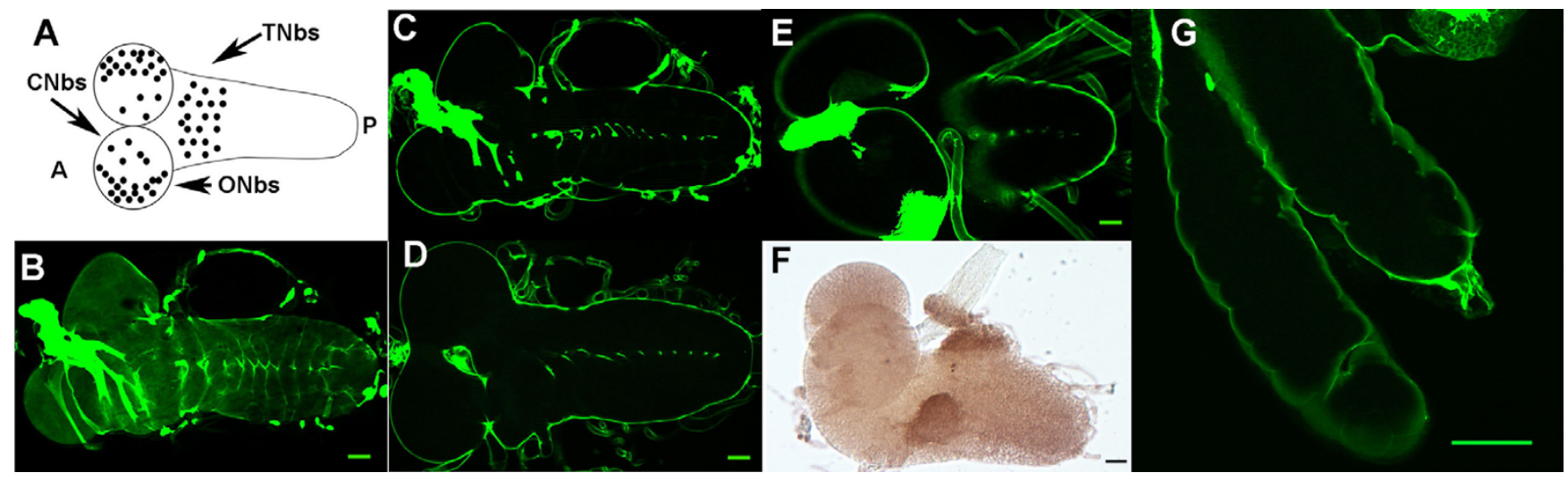

Figure 4

Localization of Trol-GFP in the larval brain. A) Schematic of neuroblast position in the larval brain. $A=$ anterior, $P=$ posterior, $\mathrm{ONbs}=$ optic lobe neuroblasts, $\mathrm{CNbs}=$ central brain neuroblasts, $\mathrm{TNbs}=$ thoracic neuroblasts. In panels B-F, Anterior is to the left, Posterior is to the right. B) Optical section of Trol-GFP brains at first instar, brain surface, scale bar indicates 10 um for both panels B and C. C) Trol-GFP localization in first instar internal section. D) Trol-GFP localization in second instar internal section, scale bar indicates $15 \mathrm{um}$. E) Trol-GFP localization in third instar internal section, scale bar indicates 25 um. F) First instar brain stained with anti-Trol antibody, showing staining over the entire surface of the brain. Scale bar indicates 10 um. G) Trol-GFP localization in internal section of third instar salivary gland. Scale bar indicates 25 um.

\section{Effects of trol mutations on larval hemocyte number}

A second system where we thought trol might have an effect on development is the production of hemocytes during larval life. A number of studies have elegantly shown that the larval lymph gland is the source of larval hemocytes [33]. In the primary lobe of the third instar lymph gland prohemocytes arise in the medullary zone while maturing hemocytes are found in the adjacent cortical zone. Hemocytes are then released into the hemolymph and are present as three types of circulating cells: plasmatocytes (95\%), lamellocytes (1-5\%) and crystal cells (rare). Each cell-type has characteristic morphology and can be easily identified under a compound microscope. Mature circulating larval hemocytes are still undergoing cell division, albeit at a low rate, as shown by staining of hemocytes with phosphohistone H3, an M phase marker [34,35]. Expression of an activated Ras $\left(\right.$ Ras $\left.^{\mathrm{v} 12}\right)$ in circulating hemocytes increases the percentage of circulating hemocytes that stain for phosphohistone $\mathrm{H} 3$ and results in a 40 -fold increase in the number of hemocytes through activation of the Ras-MAPK pathway [34]. The Ras-MAPK pathway is activated by Vascular Endothelial Growth Factor (VEGF) and Platelet Derived Growth Factor (PDGF) among others. Signaling by mammalian homologs of both growth factors has been linked to mammalian Perlecan [3]. Furthermore, studies of PDGF/VEGF receptor (PVR) in Drosophila revealed that $P V R$ is expressed in plasmatocytes and that decreased PVR function leads to increased hemocyte cell death [33]. Thus it seemed likely that mutations in trol could decrease PDGF/VEGF signaling in circulating plasmatocytes, result- ing in decreased numbers of circulating plasmatocytes in trol mutants. To address this hypothesis, we determined the relative number of circulating plasmatocytes in third instar trol $^{b 22}$ or trol $^{7}$ and sibling control larvae (Fig. 5A). Our analysis demonstrates a significant $(\mathrm{p}<0.05)$ drop in the number of plasmatocytes in trol mutant versus sibling control larvae.

\section{Trol localization and function in hemocytes}

The decrease in the number of circulating plasmatocytes in trol mutants versus controls suggested that trol might indeed function to promote Ras-MAPK signaling by PDGF/VEGF in circulating plasmatocytes. This predicts that Trol protein would be localized on these plasmatocytes. We used Trol-GFP protein trap to examine the plasmatocytes for the presence or absence of Trol protein. Fluorescence microscopy revealed that Trol-GFP is indeed found on circulating plasmatocytes in third instar larvae (Fig. 5B,C), but not in the lymph gland (data not shown).

This result is consistent with the requirement for RasMAPK activation in plasmatocytes for plasmatocyte proliferation and for PVR in plasmatocytes to avert apoptosis, and supports the hypothesis that Trol modulates PVR-RasMAPK signaling in plasmatocytes. The ETS-transcription factor pnt is a MAPK-response gene and will drive plasmatocyte proliferation [36]. Therefore we asked if trol mutant plasmatocytes show decreased levels of pnt compared to controls. Plasmatocytes were collected by bleeding third instar trol $^{\text {b22 }}$ and trol $^{7}$ mutant larvae and sibling controls, RNA was extracted and amplified, and subjected to qRT- 

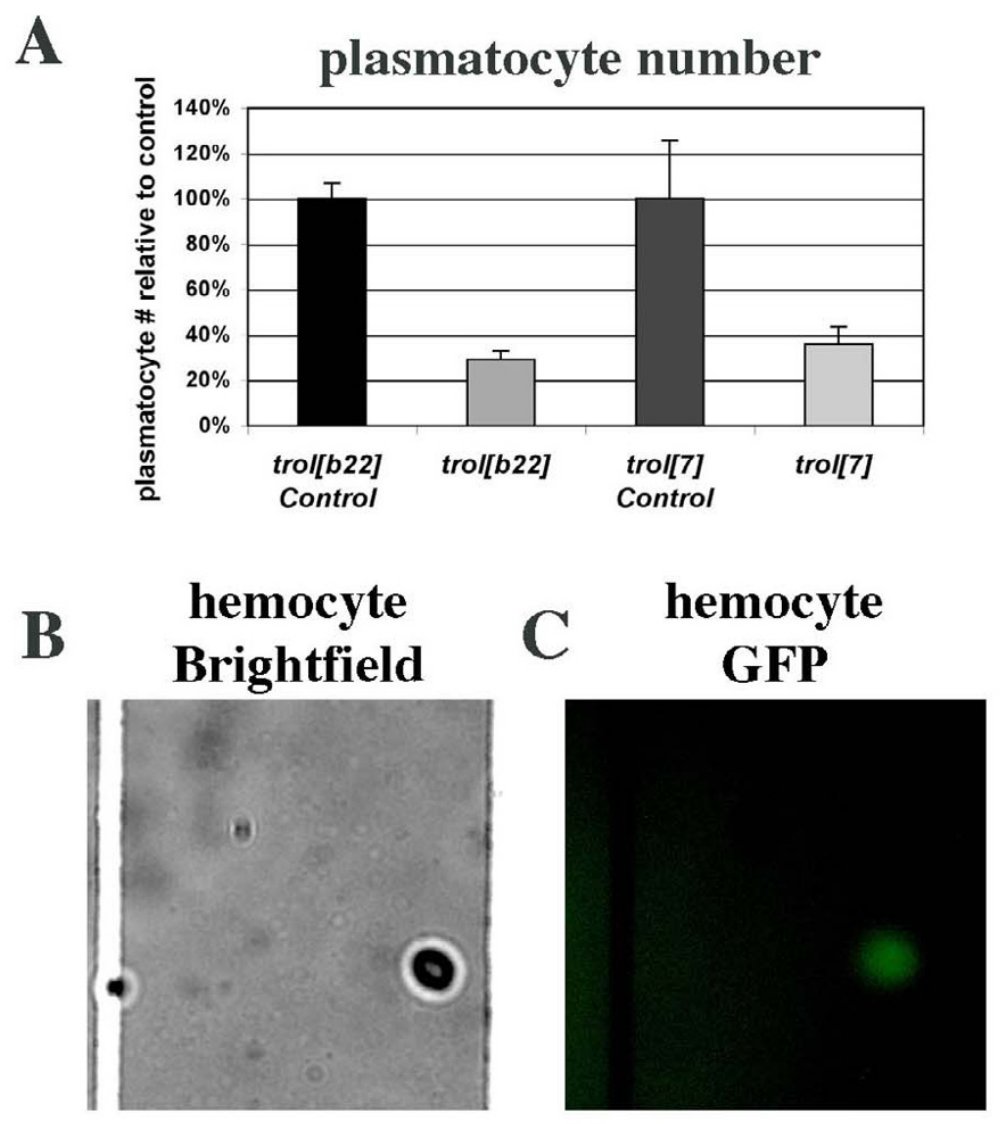

D

pnt expression level

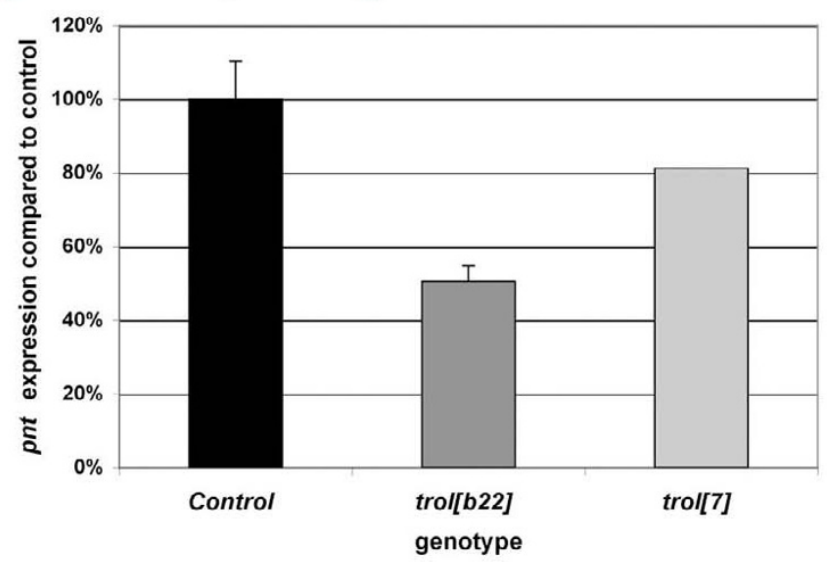

\section{Figure 5}

Mutations in trol decrease circulating plasmatocyte number and pnt expression. A) Quantification of circulating plasmatocytes in trolb22 and trol mutants compared to controls. Each sample consisted of hemolymph pooled from three third instar larvae. Five squares were counted for each sample. Each genotype was analyzed in triplicate. B) Brightfield image of plasmatocytes from Trol-GFP stock. C) Fluorescence image of plasmatocytes from Trol-GFP stock demonstrating presence of Trol on plasmatocytes. D) Expression of the VEGF/PDGF response gene pnt in trolo22 and trol mutant hemocytes compared to sibling controls by GRT-PCR. Samples of hemolymph from three third instar larvae of each genotype were pooled, RNA extracted, amplified and analyzed. All reactions were carried out in triplicate at three different template concentrations to ensure amplification was in the linear range. $\beta$-actin was used as an internal normalization control. 


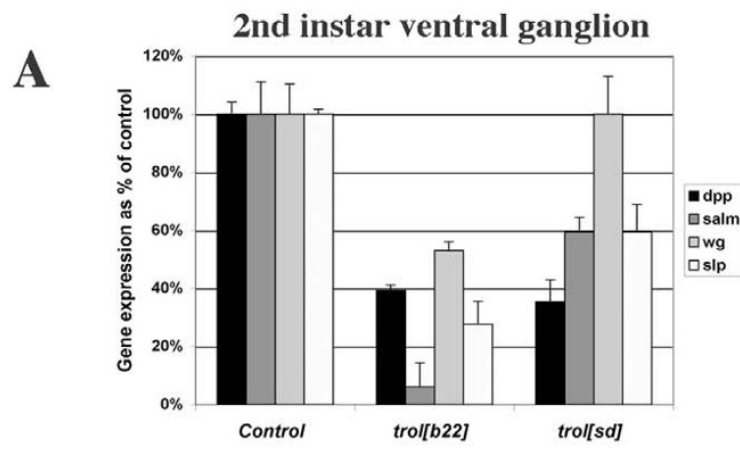

B

3rd instar brain lobes and eye dises

B

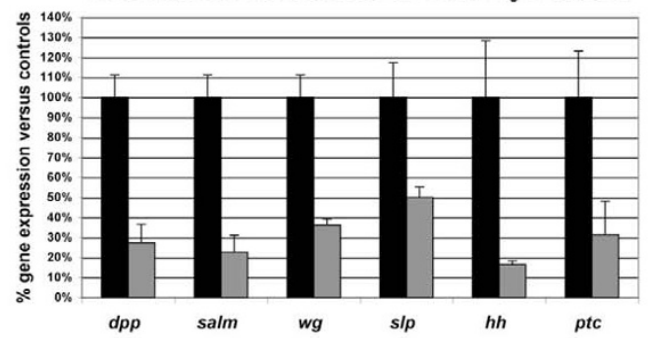

C

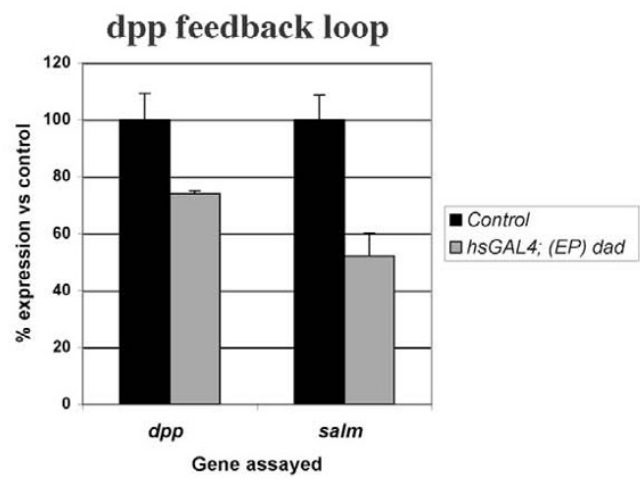

D

wg feedback loop

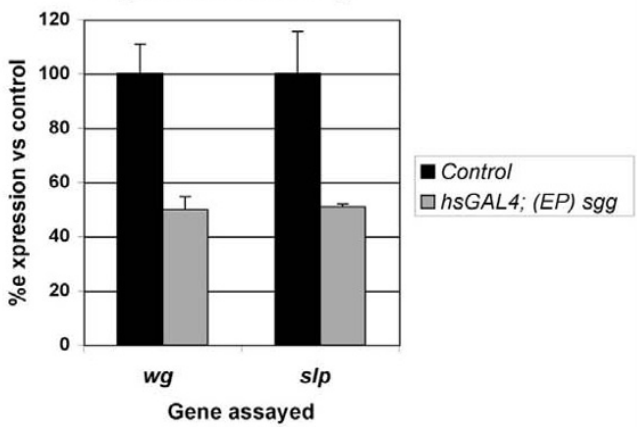

Figure 6

Dpp, Wg and Hh signaling are affected in trol mutants in second instar brains but not in third instar brain lobes/eye discs. qRT-PCR analysis of $A$ ) the expression levels of $d p p$, its response gene salm, wg and its response gene slp in the ventral ganglia of second instar larvae. Data for the Hh response gene ptc is shown in Fig. 3. B) Expression levels of $d p p$, its response gene salm, wg and its response gene $s / p, h h$ and its response gene ptc in tro/sd mutant third instar brain lobes/eye discs. C) Expression of $d p p$ and salm in hsGAL4/+; +/(EP)dad second instar ventral ganglia and hsGAL4 controls. D) Expression of wg and slp in $h s G A L 4 /+;+/(E P) s g g$ second instar ventral ganglia and hsGAL4 controls. In all panels, error bars indicate standard error. All reactions were carried out in triplicate at three different template concentrations to ensure amplification was in the linear range. $\beta$-actin was used as an internal normalization control. 
PCR analysis. qRT-PCR studies demonstrated that plasmatocytes isolated from either trolb22 or trol mutants show decreased expression of pnt compared to controls, further evidence that trol modulates Ras-MAPK signaling in plasmatocytes (Fig. 5D).

\section{Trol and other growth factor signaling pathways}

Two other growth factor signaling pathways that have been linked to HSPGs are the wingless ( $w g / \mathrm{Wnt})$ and decapentaplegic $(d p p / \mathrm{TGF} \beta)$ signaling pathways. Both of these pathways are active in the developing Drosophila eye disc and/or third instar brain along with Hh and Ras-MAPK signaling $[37,38]$. To ask if Trol might modulate the Dpp and Wg pathways we evaluated the expression of $d p p$ and $w g$ and their target genes spalt major (salm, [39]) and sloppy paired (slp, [40]), respectively, in second instar ventral ganglia and third instar brains and eye discs from trol mutant larvae by qRT-PCR (Fig. 6A-B). We also assayed expression of $h h$ and its response gene $p t c$ in third instar brains and eye discs. In the trolb22 second instar ventral ganglion we observed a significant drop in the level of both $d p p$ and $w g$ compared to controls. The trolb22 mutation also resulted in diminished signaling efficiency by both growth factors as indicated by a larger drop in the level of their response genes salm and slp compared to the ligands themselves. In contrast, the trolsd mutation decreased only $d p p$ expression, but the efficiency of both $d p p$ and $w g$ signaling was impaired. Thus in the second instar ventral gangion, wild-type function of trol appears to be required for normal signaling by $h h, b n l, d p p$ and $w g$ (Figs. 3 and $6 \mathrm{~A}$ ). The decreased expression of $d p p$ and $w g$ in trolb22 mutants and of $d p p$ in trolsd mutants may be due to secondary effects on $d p p$ and $w g$ expression caused by the changes in $\mathrm{Hh}$ and Bnl signaling in trol mutants. Alternatively, decreased expression of $d p p$ and $w g$ could be due to positive feedback between Dpp signaling and $d p p$ expression and $\mathrm{Wg}$ signaling and $w g$ expression, respectively. To test the latter possibility, we blocked Dpp signaling by overexpression of daughters against dpp (dad) [41], and assayed for $d p p$ message levels (Fig. 6C). (EP) dad females were crossed to hsGAL4 males to drive expression of $\mathrm{dad}$. Embryogenesis and first instar larval development were carried out at $18^{\circ} \mathrm{C}$ to limit expression of dad and inhibition of Dpp signaling at early stages. Upon molt to second instar, larvae were moved to $25^{\circ} \mathrm{C}$ for one hour to induce expression of $\mathrm{dad}$. Larval brains were dissected and the ventral ganglia harvested for RNA isolations. Inhibition of Dpp signaling was confirmed by analysis of salm mRNA levels. Similarly, we inhibited Wg signaling by overexpression of shaggy ( $\mathrm{sgg}$ ), and assayed for $w g$ message levels (Fig. 6D). Decreased Wg signaling was verified by analysis of slp expression levels. As shown by our qRT-PCR analysis, inhibition of Dpp signaling by over-expression of dad resulted in a drop in expression of the $d_{p p}$ ligand itself. Inhibition of Wg signaling by over-expression of $s g g$ also produced a drop in the expression of wg. As these studies were conducted in flies wild-type for trol, they eliminate the possibility that the decreased expression of $d p p$ and $w g$ in trol mutants was due solely to reduced Trolmediated signaling by $\mathrm{Hh}$ and/or Bnl. These data indicate the presence of a positive feedback loop for Dpp and Wg in the ventral ganglion.

To determine if Trol is necessary for growth factor signaling in other tissues at other stages we assayed for $d p p$, wg and $h h$ expression and activity in trolb22 and trolsd third instar brain lobes and eye discs. No significant changes in either growth factor expression or signaling were observed in trol ${ }^{b 22}$ samples (data not shown). In trolsd samples, expression of all three growth factors decreased by 65$85 \%$, as did the expression of their response genes (Fig. $6 \mathrm{~B})$. The sole exception is $w g / s l p$, where $w g$ expression decreased about $65 \%$ and slp expression decreased only about $50 \%$. These data indicate that mutations in trol do not dramatically decrease the signaling efficiency of Dpp, $\mathrm{Wg}$ or Hh in third instar brain lobes and eye discs, unlike the effect of those same trol mutations in second instar.

\section{Conclusion}

\section{trol and Drosophila development}

We have previously demonstrated that mutations in trol prevent the onset of neuroblast division in the first instar brain and that most trol mutations are lethal. Mutations in a second gene, anachronism, also affect the onset of neuroblast proliferation but in the opposite manner: in anachronism mutants, mitotically regulated neuroblasts begin cell division too early [42]. However, when a lethal trol mutation was combined with a viable allele of anachronism, the lack of neuroblast division was rescued (double mutants exhibited the anachronism phenotype of premature neuroblast division) but lethality was not [21]. This outcome suggested that trol function is required for other developmental events necessary for survival. Further analyses revealed that trol modulates $\mathrm{Hh}$ and $\mathrm{Bnl}$ signaling in the first instar brain [19]. Here we have demonstrated that trol function is required for developmental progression to third instar and for pupariation. Analogous to its function in the first instar brain, trol is required to initiate the division of a second, independent and spatially distinct population of neuroblasts in the second instar brain (Table 1, Fig. 1). This initiation of division is also dependent on Bnl and Hh signaling (Fig. 2). We have also demonstrated that the Trol protein is localized to the surface of the brain at all larval stages, which places it in close proximity to the regulated neuroblasts. This localization is consistent with our model where Trol regulates $\mathrm{Bnl}$ and Hh signaling to cells adjacent to the regulated neuroblasts by binding the growth factors directly [19]. Trol protein localization to the basal lamina is not limited to the larval brain, as TrolGFP studies also showed Trol protein in the basal lamina 
surrounding the salivary glands (Fig. 4G). trol function is not limited to the nervous system, as mutations in trol also diminish the number of circulating plasmatocytes by decreasing expression of pnt, a PVR response gene in plasmatocytes (Fig. 5). We speculate that trol may be necessary for signaling by the Drosophila PDGF and/or VEGF growth factor, just as mammalian Perlecan has been shown to function during angiogenesis [3]. Our studies of Dpp and Wg indicate a positive feedback between $d p p$ expression and Dpp signaling and $w g$ expression and $\mathrm{Wg}$ signaling in the second instar ventral ganglion. Signaling by Dpp and $\mathrm{Wg}$ is also dependent on trol in the second instar brain, but not (or very little) in the third instar brain lobes and eye discs (Fig. 6), despite the fact that Dpp and Wg signaling are taking place in those tissues. In fact, even Hh signaling appears to be independent of trol in this context. These results highlight an important concept in trol, and indeed, in proteoglycan function: that the Trol protein will be used at different times and places to regulate the signaling of different growth factors. Deciphering the role of trol in different developmental decisions will require that we examine each event individually, as trol will not necessarily mediate the same molecular mechanism each time.

\section{Involvement of HSPGs in growth factor signaling}

The requirement for heparan sulfate proteoglycans in signaling by different families of growth factors is well established [43], but what is not yet clear is why different organs and tissue types use different HSPGs to modulate these signaling pathways. One possibility is that the specific mechanism(s) through which these molecules modulate signaling activity allows for site-specific variations in the regulation of signaling activity. HSPGs with varied amino acid sequence can act in the same signaling pathway, such as Syndecan- 4 and Perlecan for FGF2 $[28,44]$ or Glypicans, Syndecan-3 and Perlecan for Hh $[19,43,45]$. Mutations that affect heparan sulfate synthesis or modification strongly affect FGF2 and Hh signaling [43]. Furthermore, Perlecan isolated from various endothelial cell sources has different binding affinities for FGF2 [46]. These data initially suggested that the protein core of the HSPG might have little to do with signaling specificity and that the main functional domain of HSPGs is concentrated in the sequence of the heparan sulfate chains.

The carbohydrate-centric view is being challenged by studies that indicate a role for the protein-protein interactions of HSPGs with growth factors and other signaling molecules. For example, expression of chimeric molecules has shown that the cytoplasmic tail of Syndecan is specifically required for FGF2 signaling in addition to its heparan sulfate chains [47]. Perlecan protein-protein interactions include the ability of Perlecan to bind growth factors and extracellular matrix molecules at various sites on its protein core. Further mechanisms that allow for differential regulation include processing of HSPGs. These studies suggest a reason for the use of a particular HSPG during an individual developmental decision - the flexibility of combining both carbohydrate-based regulation and protein-based regulation of cell-cell signaling may make a specific HSPG uniquely suited for a given situation.

In the context of combined carbohydrate and protein inputs into HSPG function, it becomes clear that a given HSPG may be expressed and function in very specific contexts that take advantage of its unique regulatory abilities. It is interesting to note that we have connected Perlecan with FGF and Hh signaling in the developing fly brain while mouse studies have shown that Perlecan knock-out mice have cerebral cortex abnormalities [6,19,21]. trol mutant larvae have decreased numbers of circulating hemocytes that are likely due to decreased Ras-MAPK signaling by VEGF/PDGF. Perlecan knock-out mice also have defects in chondrogenesis and cardiovascular development and mammalian studies have demonstrated a role for Perlecan in angiogenesis driven by FGFs, VEGF and PDGF [3]. Finally, we have shown that Perlecan is required for $\mathrm{SHH}$ signaling during human prostate cancer growth [8], which reveals a new system for the investigation of the mechanism of Perlecan action. Further analysis of the ability of HSPGs to substitute for each other in cell fate decisions and the means by which they individually regulate cell-cell communication will lead to a clearer understanding of the inputs necessary for cells to carry out a developmental or disease progression.

\section{Methods \\ Fly stocks}

Stocks of the viable trolb22 allele and the lethal $\mathrm{trol}^{4}$, $\mathrm{trol}^{7}$, trol $^{8}$ and trolsd alleles have been described previously $[19,21,22,29]$. All trol mutant stocks with the exception of trolb22 are $y$ trol $^{x} w /$ Binsn where the chromosome carrying the trol mutation is marked with $y$ to facilitate identification of $y$ trol mutant versus $\gamma^{+}$control larvae. The trol-GFP protein trap was obtained from Dr. Stephane Noselli. The $b n l^{06916}$ and $h h^{A C}$ stocks were obtained from the Bloomington stock center and used to construct $y ; b n l^{06916} /$ $T M 3 y^{+}$and $y ; h h^{A C} / T M 3 y^{+}$stocks for genetic studies.

\section{Lethal phase}

Early first instar larvae were collected and placed on apple juice plates with yeast. Each plate initially had 50 mutant or control animals per plate, segregated to prevent competition between mutant and wildtype siblings. Two plates of each genotype were examined. The number and stage of larvae still present on each plate were assayed every 24 hours and the survivors transferred to a fresh plate. Since none of the trol mutants with the exception of trolb22 pro- 
duce viable adults, individual animals were followed only until pupariation.

\section{Developmental staging}

Developmental synchronization was carried out as previously described $[19,21,23,48]$. Flies were allowed to lay eggs on apple juice agar plates with fresh yeast overnight or for about 24 hours. For staging of synchronized first instar larvae, the plate was first cleared of any larvae and newly hatched larvae collected in one hour windows and placed on new apple juice plates with yeast at $25^{\circ} \mathrm{C}$ for aging. For staging of second instar larvae, late first instar larvae were placed on fresh apple juice plates with yeast. Newly molted second instars were collected in one hour windows and placed on apple juice plates with yeast at $25^{\circ} \mathrm{C}$ for aging or dissected immediately.

\section{Proliferation assay}

BrdU assays were carried out as previously described $[19,21,23,48]$. Briefly, animals were fed BrdU-containing artificial medium for one hour, dissected in PBST and fixed with Histochoice (Amresco) for 10 minutes. Brain samples were denatured in PBST-HCl for 30 minutes, washed and blocked in PBNT for one hour. Primary antiBrdU antibody (Becton-Dickinson) was added at 1:200 overnight at $4{ }^{\circ} \mathrm{C}$. Samples were washed and incubated with HRP-conjugated secondary antibody at 1:400 for 24 hours at room temperature. Signal was developed using a DAB substrate (Sigma).

\section{Larval hemocyte assay}

Hemocytes from three third instar larvae were harvested using a Pasteur pipette pulled to generate a capillary end, pooled and counted on a standard hemacytometer slide. Five 16-square regions were counted for each pooled sample. Three replicates were assayed for each genotype.

\section{Quantitative RealTime PCR}

Whole first instar brains or ventral ganglia dissected from the brains of second instar larvae were used for RNA isolation. For first instar brain samples, total RNA was isolated using Trizol (Invitrogen) following manufacturer's directions. Samples were DNAsed and reverse transcribed using oligo dT primers. The resulting CDNA was used to perform quantitative Real Time PCR with SYBR Green dye. For ventral ganglia isolated during second instar RNA was extracted and the sequences amplified as described in $[49,50]$. Hemocyte studies were carried out on pooled hemolymph from three third instar larvae per sample. RNA was extracted and amplified as for ventral ganglia. All qRT-PCR reactions were carried out in triplicate at three different template concentrations to ensure that we were within linear template range. Primer sequences are available upon request. $\beta$-actin expression was used as an internal control. Data were analyzed using the delta-delta calculation method to yield fold change compared to controls.

\section{Statistics}

Determination of significance was accomplished by use of Student's t test or ANOVA, depending on the design of the study.

\section{List of Abbreviations}

Bnl: Branchless

BrdU: 5-Bromodeoxyuridine

dpp: decapentaplegic

FGF: Fibroblast Growth Factor

GFP: Green Fluorescent Protein

Hh: Hedgehog

HSPG: Heparan Sulfate Proteoglycan

PDGF: Platelet Derived Growth Factor

PVR: PDGF- and VEGF-Receptor Related

qRT-PCR: quantitative Real Time PCR

SHH: Sonic Hedgehog

TGF $\beta$ : Transforming Growth Factors

TNb: Thoracic neuroblast

VEGF: Vascular Endothelial Growth Factor

wg: wingless

\section{Authors' contributions}

JL carried out all of the TNb genetic and molecular analyses, localization of Trol-GFP in the larval brain, hemocyte counting and qRT-PCR in hemocytes. JL also oversaw the trol developmental/lethality phenotypic analyses.

PH carried out the trol developmental/lethality analyses and participated in the trol $\mathrm{TNb}$ studies.

$\mathrm{AB}$ oversaw the trol hemocyte counting studies.

MC participated in the trol hemocyte counting studies.

TP carried out the qRT-PCR analyses of first instar trol larval brains. 
YP carried out the BrdU study of the trol7 first instar neuroblast phenotype.

SD conceived of the studies, contributed to the experimental design and interpretation of all studies and wrote the manuscript.

\section{All authors have read and approved the final manuscript.}

\section{Acknowledgements}

The authors would like to thank past and present members of the Datta lab, anonymous reviewers and Drs. Subhabrata Sanyal and Vlad Panin for helpful discussions, the Bloomington stock center and Dr. Stephane Noselli for fly stocks, Dr. Larry Ringer for help with statistical analyses and Dr. Shubha Govind for advice on hemocyte counting. Alyssa Rosenbloom, Bryan Tackett, Rachel Miller, Megan Carrier and Allegra Lamison provided technical support. This work was supported by NIH grant NS 036737-04 to S. D.

\section{References}

I. Lin X: Functions of heparan sulfate proteoglycans in cell signaling during development. Development 2004, I 3 | (24):6009-602 I.

2. Datta S, Pierce M, Datta MW: Perlecan signaling: helping hedgehog stimulate prostate cancer growth. Int J Biochem Cell Biol 2006, 38(I I): |855-|86I.

3. lozzo RV: Basement membrane proteoglycans: from cellar to ceiling. Nat Rev Mol Cell Biol 2005, 6(8):646-656.

4. Arikawa-Hirasawa E, Watanabe H, Takami H, Hassell JR, Yamada Y: Perlecan is essential for cartilage and cephalic development. Nat Genet 1999, 23(3):354-358.

5. Costell M, Carmona R, Gustafsson E, Gonzalez-Iriarte M, Fassler R, Munoz-Chapuli R: Hyperplastic conotruncal endocardial cushions and transposition of great arteries in perlecan-null mice. Circ Res 2002, 91 (2): I58-164.

6. Costell M, Gustafsson E, Aszodi A, Morgelin M, Bloch W, Hunziker E, Addicks K, Timpl R, Fassler R: Perlecan maintains the integrity of cartilage and some basement membranes. J Cell Biol 1999, I 47(5): I I09-I I 22.

7. Gonzalez-Iriarte M, Carmona R, Perez-Pomares JM, Macias D, Costell $M$, Munoz-Chapuli R: Development of the coronary arteries in a murine model of transposition of great arteries. J Mol Cell Cardiol 2003, 35(7):795-802.

8. Datta MW, Hernandez AM, Schlicht MJ, Kahler AJ, Degueme AM, Dhir R, Shah RB, Farach-Carson MC, Barrett A, Datta S: Perlecan, a candidate gene for the CABP locus, regulates prostate cancer cell growth via the Sonic Hedgehog pathway. Mol Cancer 2006, 5(I):9.

9. Mongiat M, Sweeney SM, San Antonio JD, Fu J, lozzo RV: Endorepellin, a novel inhibitor of angiogenesis derived from the $C$ terminus of perlecan. J Biol Chem 2003, 278(6):4238-4249.

10. Sharma B, Handler M, Eichstetter I, Whitelock JM, Nugent MA, lozzo RV: Antisense targeting of perlecan blocks tumor growth and angiogenesis in vivo. J Clin Invest 1998, I 02(8): I 599- I608.

II. Zhou Z, Wang J, Cao R, Morita H, Soininen R, Chan KM, Liu B, Cao $Y$, Tryggvason K: Impaired angiogenesis, delayed wound healing and retarded tumor growth in perlecan heparan sulfatedeficient mice. Cancer Res 2004, 64(1 4):4699-4702.

12. lozzo RV, San Antonio JD: Heparan sulfate proteoglycans: heavy hitters in the angiogenesis arena. J Clin Invest 200I, I 08(3):349-355

13. Arikawa-Hirasawa E, Le AH, Nishino I, Nonaka I, Ho NC, Francomano CA, Govindraj P, Hassell JR, Devaney JM, Spranger J, Stevenson RE, lannaccone S, Dalakas MC, Yamada Y: Structural and functional mutations of the perlecan gene cause SchwartzJampel syndrome, with myotonic myopathy and chondrodysplasia. Am J Hum Genet 2002, 70(5): I 368-I375.

14. Arikawa-Hirasawa E, Wilcox WR, Le AH, Silverman N, Govindraj P, Hassell JR, Yamada Y: Dyssegmental dysplasia, Silverman-
Handmaker type, is caused by functional null mutations of the perlecan gene. Nat Genet 200I, 27(4):43I-434

15. Nicole S, Davoine CS, Topaloglu H, Cattolico L, Barral D, Beighton P, Hamida CB, Hammouda H, Cruaud C, White PS, Samson D, Urtizberea JA, Lehmann-Horn F, Weissenbach J, Hentati F, Fontaine B: Perlecan, the major proteoglycan of basement membranes, is altered in patients with Schwartz-Jampel syndrome (chondrodystrophic myotonia). Nat Genet 2000, 26(4):480-483.

16. Rogalski TM, Williams BD, Mullen GP, Moerman DG: Products of the unc-52 gene in Caenorhabditis elegans are homologous to the core protein of the mammalian basement membrane heparan sulfate proteoglycan. Genes Dev |993, 7(8): |47|-|484.

17. Brenner S: The genetics of Caenorhabditis elegans. Genetics 1974, 77(I):71-94.

18. Rogalski TM, Mullen GP, Bush JA, Gilchrist EJ, Moerman DG: UNC52/perlecan isoform diversity and function in Caenorhabditis elegans. Biochem Soc Trans 200I, 29(Pt 2): I7I-I76.

19. Park Y, Rangel C, Reynolds MM, Caldwell MC, Johns M, Nayak M, Welsh CJ, McDermott S, Datta D: Drosophila Perlecan modulates FGF and Hedgehog signals to activate neural stem cell division. Dev Biol 2003, 253:247-257.

20. Voigt A, Pflanz R, Schafer U, Jackle H: Perlecan participates in proliferation activation of quiescent Drosophila neuroblasts. Dev Dyn 2002, 224(4):403-4I 2

21. Datta S: Control of proliferation activation in quiescent neuroblasts of the Drosophila central nervous system. Development 1995, I 2 I(4): I I73-I I 82.

22. Datta S, Kankel DR: I(I)trol and I(I)devl, loci affecting the development of the adult central nervous system in Drosophila melanogaster. Genetics 1992, I 30:523-537

23. Caldwell MC, Datta S: Expression of cyclin E or DP/E2F rescues the G I arrest of trol mutant neuroblasts in the Drosophila larval central nervous system. Mechanisms of Development I998, 79(I-2): |2|-| 30.

24. Park $\mathrm{Y}, \mathrm{Ng} \mathrm{C}$, Datta $\mathrm{S}$ : Induction of string rescues the neuroblast proliferation defect in trol mutant animals. Genesis 2003 , 36(4): 187-195.

25. Schneider M, Khalil AA, Poulton J, Castillejo-Lopez C, Egger-Adam D, Wodarz A, Deng WM, Baumgartner S: Perlecan and Dystroglycan act at the basal side of the Drosophila follicular epithelium to maintain epithelial organization. Development 2006, I33( I9):3805-38। 5 .

26. Truman JW, Bate M: Spatial and temporal patterns of neurogenesis in the central nervous system of Drosophila melanogaster. Developmental Biology |988, I 25:|45-|57.

27. Prokop A, Technau GM: The origin of postembryonic neuroblasts in the ventral nerve chord of Drosophila melanogaster. Development 1991, I I I:79-88.

28. Aviezer D, lozzo RV, Noonan DM, Yayon A: Suppression of autocrine and paracrine functions of basic fibroblast growth factor by stable expression of perlecan antisense cDNA. Mol Cell Biol 1997, I7(4): 1938-1946.

29. Park Y, Fujioka M, Jaynes JB, Datta S: Drosophila homeobox gene eve enhances trol, an activator of neuroblast proliferation in the larval CNS. Developmental Genetics 1998, 23(3):247-257.

30. Friedrich MV Schneider M, Timpl R, Baumgartner S. Perlecan domain $V$ of Drosophila melanogaster. Sequence, recombinant analysis and tissue expression. Eur J Biochem 2000, 267( I I):3|49-3 I59.

31. Medioni C, Noselli S: Dynamics of the basement membrane in invasive epithelial clusters in Drosophila. Development 2005, I32(13):3069-3077.

32. White K, Kankel DR: Patterns of cell division and cell movement in the formation of the imaginal nervous system in Drosophila melanogaster. Developmental Biology 1978, 65:296-321

33. Evans C], Hartenstein V, Banerjee U: Thicker than blood: conserved mechanisms in Drosophila and vertebrate hematopoiesis. Dev Cell 2003, 5(5):673-690.

34. Asha H, Nagy I, Kovacs G, Stetson D, Ando I, Dearolf CR: Analysis of Ras-induced overproliferation in Drosophila hemocytes. Genetics 2003, I 63(I):203-215.

35. Qiu P, Pan PC, Govind S: A role for the Drosophila Toll/Cactus pathway in larval hematopoiesis. Development 1998 , I 25( I 0): | 909-1920. 
36. Zettervall C], Anderl I, Williams MJ, Palmer R, Kurucz E, Ando I, Hultmark $D$ : A directed screen for genes involved in Drosophila blood cell activation. Proc Natl Acad Sci U S A 2004, I0I(39): |4|92-|4|97.

37. Kaphingst K, Kunes S: Pattern formation in the visual centers of the Drosophila brain: wingless acts via decapentaplegic to specify the dorsoventral axis. Cell 1994, 78(3):437-448.

38. Silver S], Rebay I: Signaling circuitries in development: insights from the retinal determination gene network. Development 2005, I32(1):3-13.

39. de Celis JF, Barrio R: Function of the spalt/spalt-related gene complex in positioning the veins in the Drosophila wing. Mech Dev 2000, 9 I (I-2):3|-4I.

40. Bhat KM, van Beers EH, Bhat P: Sloppy paired acts as the downstream target of wingless in the Drosophila CNS and interaction between sloppy paired and gooseberry inhibits sloppy paired during neurogenesis. Development 2000, I 27(3):655-665.

41. Tsuneizumi K, Nakayama T, Kamoshida Y, Kornberg TB, Christian JL, Tabata T: Daughters against dpp modulates dpp organizing activity in Drosophila wing development. Nature 1997, 389(665I):627-63I.

42. Ebens AJ, Garren H, Cheyette BNR, Zipursky SL: The Drosophila anachronism locus: A glycoprotein secreted by glia inhibits neuroblast proliferation. Cell 1993, 74(I):15-28.

43. Hacker U, Nybakken K, Perrimon N: Heparan sulphate proteoglycans: the sweet side of development. Nat Rev Mol Cell Biol 2005, 6(7):530-54I.

44. Tkachenko E, Rhodes JM, Simons M: Syndecans: new kids on the signaling block. Circ Res 2005, 96(5):488-500.

45. Shimo T, Gentili C, Iwamoto M, Wu C, Koyama E, Pacifici M: Indian hedgehog and syndecans- 3 coregulate chondrocyte proliferation and function during chick limb skeletogenesis. Dev Dyn 2004, 229(3):607-617.

46. Knox S, Merry C, Stringer S, Melrose J, Whitelock J: Not all perlecans are created equal: interactions with fibroblast growth factor (FGF) 2 and FGF receptors. J Biol Chem 2002, 277( I 7): | 4657-| 4665.

47. Volk R, Schwartz JJ, Li J, Rosenberg RD, Simons M: The role of syndecan cytoplasmic domain in basic fibroblast growth factordependent signal transduction. I Biol Chem 1999, 274(34):244I7-24424.

48. Park Y, Fujioka M, Kobayashi M, Jaynes JB, Datta S: even skipped is required to produce a trans-acting signal for larval neuroblast proliferation that can be mimicked by ecdysone. Development 200I, I 28(10):1899-1909.

49. Klebes A, Biehs B, Cifuentes F, Kornberg TB: Expression profiling of Drosophila imaginal discs. Genome Biol 2002, 3(8): RESEARCH0038.

50. Klebes A, Sustar A, Kechris K, Li H, Schubiger G, Kornberg TB: Regulation of cellular plasticity in Drosophila imaginal disc cells by the Polycomb group, trithorax group and lama genes. Development 2005, I32(16):3753-3765.

Publish with Bio Med Central and every scientist can read your work free of charge

"BioMed Central will be the most significant development for disseminating the results of biomedical research in our lifetime. "

Sir Paul Nurse, Cancer Research UK

Your research papers will be:

- available free of charge to the entire biomedical community

- peer reviewed and published immediately upon acceptance

- cited in PubMed and archived on PubMed Central

- yours - you keep the copyright

Submit your manuscript here:

http://www.biomedcentral.com/info/publishing_adv.asp
BioMedcentral 